\title{
Novel diaphragmatic reconstruction technique for recurrent diaphragmatic hernia: a case report
}

Teddy HY Wong, MRCS, Simon CY Chow, FRCS, Peter SY Yu, MRCS, Jacky YK Ho, FRCS, Rainbow WH Lau, FRCS, Innes YP Wan, FRCS, Randolph HL Wong *, FRCS

Division of Cardiothoracic Surgery, Department of Surgery, Prince of Wales Hospital, Hong Kong

*Corresponding author: wonhl1@surgery.cuhk.edu.hk

Hong Kong Med J 2021;27:290-2

https://doi.org/10.12809/hkmj208790

\section{Case report}

A 51-year-old lady with a history of congenital diaphragmatic hernia repair during infancy presented to the emergency department with increasing abdominal pain and repeated vomiting. Posteroanterior chest plain radiograph revealed dilated small bowel loops in the right thoracic cavity. Computed tomography scan revealed two closely related small bowel loops trapped within a narrow hernia neck orifice across the diaphragm, suggestive of closed loop intestinal obstruction (Fig 1). Blood test results revealed metabolic acidosis and elevated lactate level.

Emergency laparotomy via a subcostal incision revealed a right-sided large diaphragmatic defect with bowel herniating into the thoracic cavity. Transabdominal reduction of the bowel was difficult owing to adhesion of bowel to the lung so an additional posterolateral thoracotomy was performed by a cardiothoracic team. Adhesiolysis was performed and the small bowel freed and reduced to the abdominal cavity via the transthoracic approach. Examination through the thoracotomy revealed minimal residual diaphragmatic tissue (Fig 2a). Primary closure was not possible and repair with a patch posed a technical challenge in the absence of sufficient residual diaphragmatic tissue to enable secure anchorage. For the anterolateral portion, multiple pledgetted $3-\mathrm{O}$ sutures were passed through the intercostal space for anchorage (Fig 2b). A neo-diaphragm was reconstructed using a porcine dermal collagen implant of size $150 \mathrm{~mm} \times 200 \mathrm{~mm} \times 1 \mathrm{~mm}$ (Permacol ${ }^{\mathrm{TM}}$; Medtronic, Minneapolis [MN], United States). The patch was then parachuted down to cover the defect and secured. A 4-cm flutter-valve patch was directed downwards and medially to avoid suturing and injury to the oesophagus and inferior vena cava (Fig 2c). We believe this design permits peristalsis of oesophageal content without causing stricture while also preventing future intestinal herniation.

Postoperatively the patient was prescribed total parenteral nutrition for 10 days and gradually tolerated a normal diet. A computed tomography scan at 10 days after surgery confirmed an intact

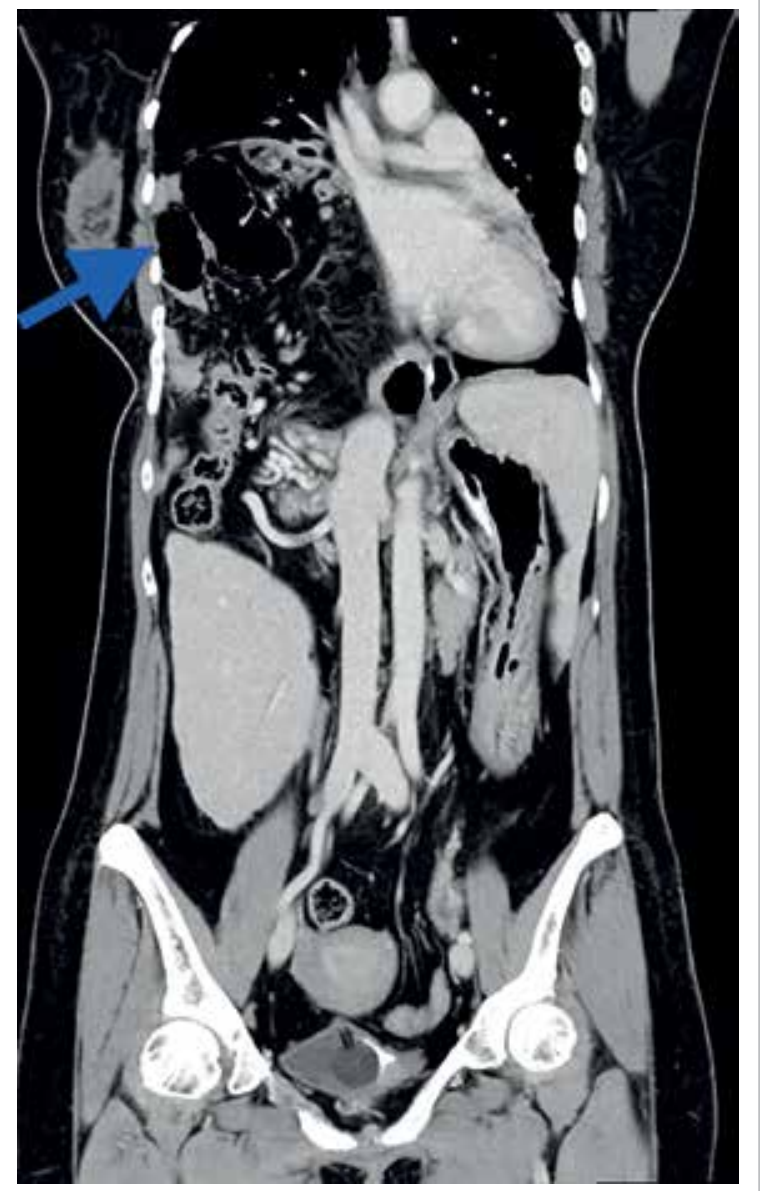

FIG I. Computed tomography showing large right diaphragmatic hernial defect (blue arrow)

neo-diaphragm with no recurrence of hernia. The patient was discharged home 13 days after surgery. She was well at follow-up examinations at 1 month and 12 months after surgery. Chest plain radiograph confirmed no recurrence of hernia (Fig 3).

\section{Discussion}

Diaphragmatic hernia is a rare but serious condition associated with respiratory and gastrointestinal complications and increased mortality. 


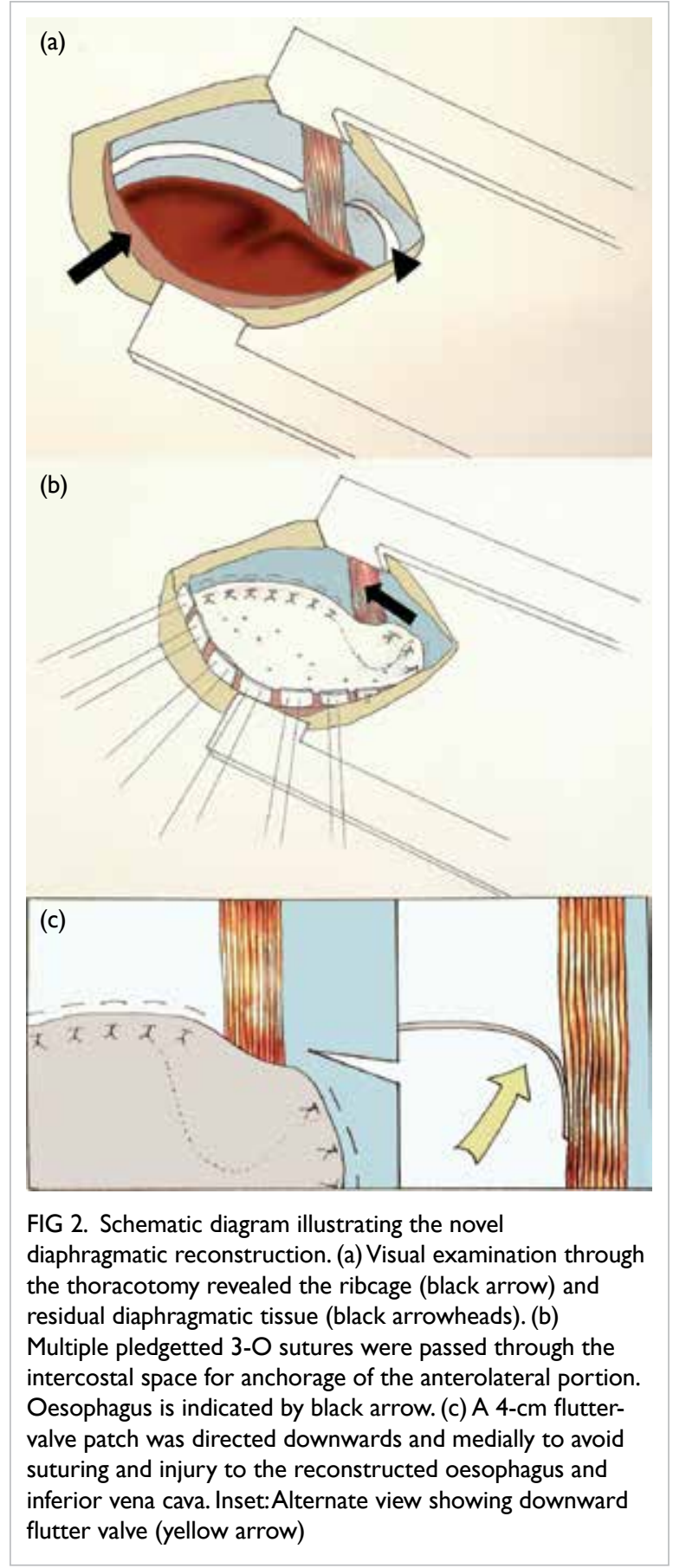

Surgical repair is the gold standard treatment to prevent complications. However, patients with a previously repaired congenital diaphragmatic hernia are more prone to respiratory complications during childhood and occasionally develop recurrences. ${ }^{1}$ However, the long-term success rate of repair is good with patients surviving well into adulthood with a normal life expectancy.

Surgical options for repair include a primary repair and a patch repair. A patch repair is associated with higher risks of complications and recurrence. ${ }^{2}$ However, for a hernia with large defects in which a

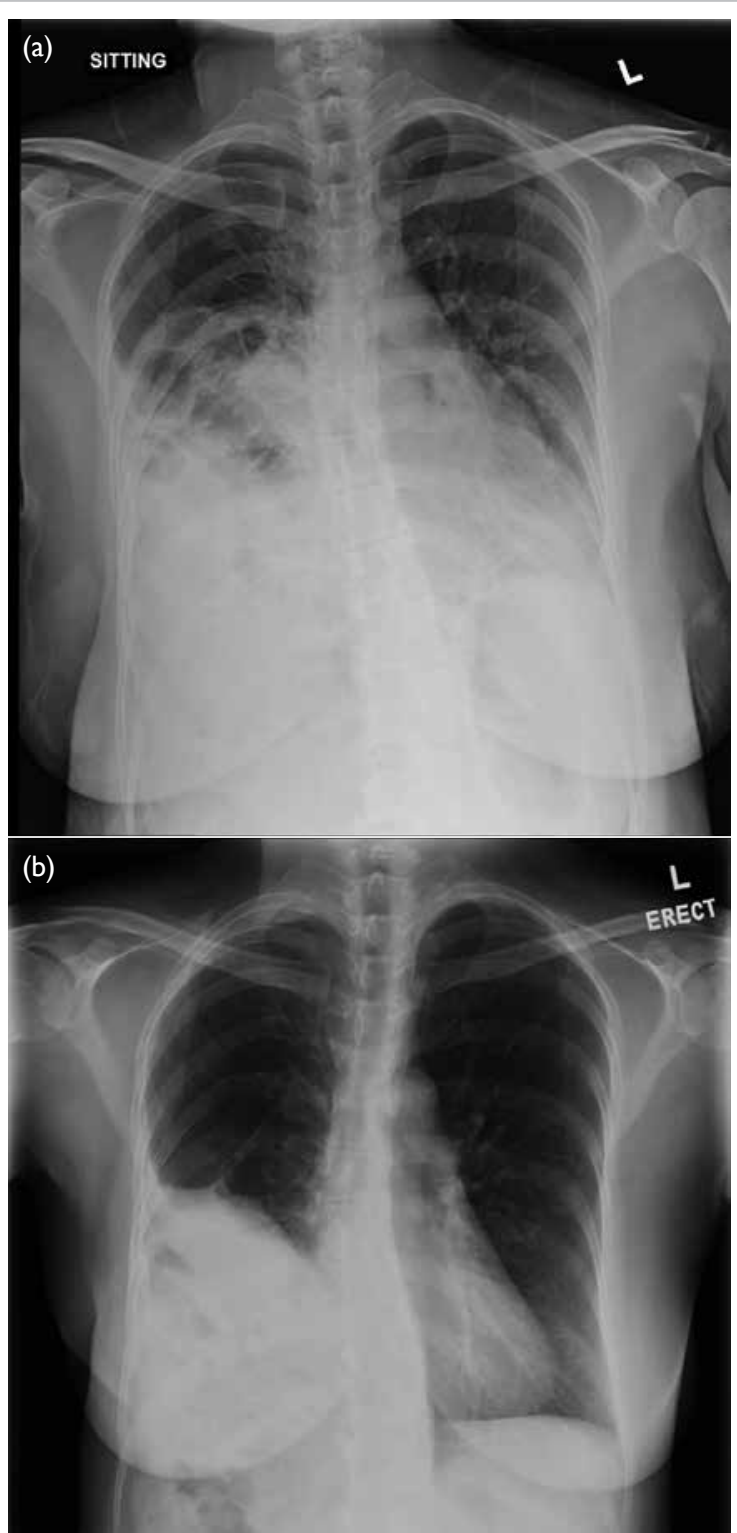

FIG 3. Chest plain radiographs of the patient (a) before and (b) after surgical repair of the diaphragmatic hernia with neo-diaphragm

primary repair is not possible, repair with surgical mesh (xenograft or synthetic) is an effective and safe method. ${ }^{3}$ In conventional patch repair, the hernia sac is repositioned and resected. The hernia defect is sized and the Permacol trimmed to cover the defect. The flap is then fixed to the remaining rim of diaphragmatic tissue with absorbable sutures. ${ }^{4}$ The lack of an adequate rim of tissue in our patient presented a unique challenge for construction of the neo-diaphragm relative to most instances of diaphragmatic hernia where a remnant of diaphragmatic tissue is present for anchorage. Although the pleura and adjacent chest wall offer good support for anchoring the 
neo-diaphragm posteriorly and anterolaterally, there is often a lack of strong tissue medially with consequent substantial risk of inadvertent injury to the oesophagus if sutures are placed too deeply. Travers et $\mathrm{al}^{5}$ reported an incidence of oesophageal injury up to $3.9 \%$ in their series of surgical repair of para-oesophageal hernia using porcine dermal collagen biologic mesh (Permacol). Innovative designs in the creation of the neo-diaphragm have been reported, but most studies have been in paediatric patients. ${ }^{6}$ The technique described in this case report is novel and has not been reported elsewhere. The design of a flutter valve mechanism in the reconstruction of the medial aspect of the neo-diaphragm serves to create a tension-free repair near the mediastinum and oesophagus while also creating a seal to separate the pleural cavity from the abdominal contents. No sutures were applied near the oesophagus or the medial side of the neo-diaphragm. This technique avoids inadvertent visceral injury and is currently our preferred technique of neodiaphragm construction in patients with a large diaphragmatic defect and insufficient rim.

In our case, we utilised the acellular dermal matrix Permacol for hernia repair. Permacol is a cross-linked graft comprised of acellular collagen matrix. Compared with other collagen-based implants, it offers long-lasting dimensional stability and avoids loss of tensile strength as well as increased tissue laxity resulting in lower rates of recurrence. Acellular dermal matrix is a developing technology and studies have shown promising results for its efficacy. ${ }^{7}$

In conclusion, repair of diaphragmatic hernia with a xenograft composed of dermal collagen implant and a medial flutter-valve design is safe and effective. It avoids inadvertent injury to mediastinal structures while allowing satisfactory prevention of recurrence of diaphragmatic herniation of intestines.

\section{Author contributions}

Concept or design: All authors.

Acquisition of data: All authors.

Analysis or interpretation of data: All authors.

Drafting of the manuscript: THY Wong, SCY Chow, RHL Wong.
Critical revision of the manuscript for important intellectual content: All authors.

All authors had full access to the data, contributed to the study, approved the final version for publication, and take responsibility for its accuracy and integrity.

\section{Conflicts of interest}

All authors have disclosed no conflicts of interest.

\section{Funding/support}

This study received no specific grant from any funding agency in the public, commercial, or not-for-profit sectors.

\section{Ethics approval}

The patient was treated in accordance with the tenets of the Declaration of Helsinki. The patient provided written informed consent for all treatments and procedures and consent for publication.

\section{References}

1. Jancelewicz T, Chiang M, Oliveira C, Chiu PP. Late surgical outcomes among congenital diaphragmatic hernia (CDH) patients: why long-term follow-up with surgeons is recommended. J Pediatr Surg 2013;48:935-41.

2. Jancelewicz T, Vu LT, Keller RL, et al. Long-term surgical outcomes in congenital diaphragmatic hernia: observations from a single institution. J Pediatr Surg 2010;45:155-60.

3. Kuhn R, Schubert D, Wolff St, Marusch F, Lippert H, Pross M. Repair of diaphragmatic rupture by laparoscopic implantation of a polytetrafluoroethylene patch. Surg Endosc 2002;16:1495.

4. Lingohr P, Galetin T, Vestweber B, Matthaei H, Kalff JC, Vestweber $\mathrm{KH}$. Conventional mesh repair of a giant iatrogenic bilateral diaphragmatic hernia with an enterothorax. Int Med Case Rep J 2014;7:23-5.

5. Travers HC, Brewer JO, Smart NJ, Wajed SA. Diaphragmatic crural augmentation utilising cross-linked porcine dermal collagen biologic mesh (Permacol) in the repair of large and complex para-oesophageal herniation: a retrospective cohort study. Hernia 2016;20:311-20.

6. Loff $\mathrm{S}$, Wirth $\mathrm{H}$, Jester I, et al. Implantation of a coneshaped double-fixed patch increases abdominal space and prevents recurrence of large defects in congenital diaphragmatic hernia. J Pediatr Surg 2005;40:1701-5.

7. Buinewicz B, Rosen B. Acellular cadaveric dermis (AlloDerm): a new alternative for abdominal hernia repair. Ann Plast Surg 2004;52:188-94. 